Revue d'études américaines. American Studies Journal

\title{
Quand le mieux est l'ennemi du bien : David Ross Brower, la militance environnementaliste et la prospérité matérielle
}

\author{
Jean-Daniel Collomb
}

\section{OpenEdition}

Journals

\section{Édition électronique}

URL : https://journals.openedition.org/transatlantica/10350

DOI : $10.4000 /$ transatlantica. 10350

ISSN : 1765-2766

\section{Éditeur}

Association française d'Etudes Américaines (AFEA)

\section{Référence électronique}

Jean-Daniel Collomb, «Quand le mieux est l'ennemi du bien : David Ross Brower, la militance environnementaliste et la prospérité matérielle », Transatlantica [En ligne], 2 | 2017, mis en ligne le 19 avril 2019, consulté le 01 février 2023. URL : http://journals.openedition.org/transatlantica/10350 : DOI : https://doi.org/10.4000/transatlantica.10350

Ce document a été généré automatiquement le 1 février 2023.

\section{cc) (†)}

Creative Commons - Attribution - Pas d'Utilisation Commerciale - Pas de Modification 4.0 International - CC BY-NC-ND 4.0

https://creativecommons.org/licenses/by-nc-nd/4.0/ 


\title{
Quand le mieux est l'ennemi du bien : David Ross Brower, la militance environnementaliste et la prospérité matérielle
}

\author{
Jean-Daniel Collomb
}

\section{Introduction}

1 Depuis les années 1960, le mouvement environnementaliste s'est forgé une place non négligeable dans le paysage politique américain, notamment au sein de la mouvance qui soutient le Parti démocrate. Or il convient de noter que l'âge d'or du mouvement environnementaliste aux États-Unis a coïncidé avec une période de forte expansion économique durant les décennies qui ont suivi la Seconde Guerre mondiale. Cette coïncidence mérite d'être interrogée, ne serait-ce que parce que la croissance économique et la distribution élargie des fruits de la prospérité sont souvent perçues par nombre d'environnementalistes comme des sources majeures de destruction environnementale et de pollution.

2 Cet article porte donc sur la tension entre l'intérêt grandissant pour les questions environnementales dans les sociétés riches et économiquement développées et la méfiance à l'égard de la croissance économique, souvent accompagnée d'un éloge de la frugalité, contenue dans nombre de prises de position environnementalistes. Afin d'éclairer cette tension, cette analyse s'appuie sur une étude approfondie de l'engagement militant de David Ross Brower des années 1950 aux années 1970. Brower est l'un des militants environnementalistes les plus influents de l'après-guerre, notamment au sein du Sierra Club, qu'il a totalement transformé, et de l'organisation Friends of the Earth, qu'il a fondée. Il incarne parfaitement la transition entre le conservationnisme du début $\mathrm{du} \mathrm{xx}^{\mathrm{e}}$ siècle et l'environnementalisme contemporain. 
3 David Brower profite habilement des conditions nouvelles créées par la société d'abondance, c'est-à-dire d'un climat socio-économique très favorable à une prise en compte de la qualité de vie et des questions environnementales. Dans le même temps, il instruit le procès de la consommation de masse, du culte de la croissance et de l'expansion démographique. Son succès militant et les nombreuses victoires qu'il remporte dépendent de conditions socio-économiques qu'il déplore et contre lesquelles il milite; la distribution de plus en plus large de l'abondance matérielle est donc à l'origine à la fois de l'éveil environnementaliste de nombreux Américains et des problèmes environnementaux qui provoquent cet éveil. Bien évidemment, tout militantisme dépend inéluctablement d'un état de fait jugé néfaste. Ainsi, pas de mouvement pour les droits civiques sans discrimination raciale. À terme, ces mouvements remportent une victoire décisive lorsque l'objet de leur mobilisation a disparu. À y regarder de plus près, néanmoins, le militantisme environnementaliste dans la société d'abondance ne semble pas obéir exactement au même schéma. Certes, l'abondance matérielle suscite la contestation de ses effets destructeurs et la remise en cause de sa légitimité ; mais lorsque l'abondance matérielle est effectivement remise en cause, comme lors de la crise énergétique des années 1970, l'opinion risque de se détourner du souci de protection de l'environnement, ce qui, in fine, peut mettre en péril les acquis environnementalistes. Si l'abondance est la condition sine qua non des victoires environnementalistes, sa remise en cause peut aboutir, paradoxalement, à un désintérêt pour la cause environnementaliste.

4 Le présent article répond à un objectif double. Il éclaire, dans la première partie, le lien de corrélation entre prospérité économique et vigueur de la militance environnementaliste, puis étudie les conséquences de cette corrélation et les impasses auxquelles elle est susceptible de conduire: comment formuler une critique substantielle et efficace de la croissance et du consumérisme tout en étant obligé de s'appuyer sur une base militante que l'opulence matérielle a fait naître ? La deuxième partie retrace le parcours militant et les succès de David Brower dans le contexte de la société d'abondance. Enfin, la troisième partie contient une analyse des tensions inhérentes au déploiement d'une rhétorique de l'austérité matérielle dans le cadre d'une société d'abondance. Cette étude s'appuie principalement sur l'étude des archives de David Ross Brower rassemblées à la bibliothèque Bancroft de l'université de Californie à Berkeley.

\section{Sociétés post-matérialistes et protection de l'environnement}

5 Selon les politologues Ronald Inglehart et Christian Welzel, l'enrichissement exceptionnel de la plupart des pays occidentaux à partir des révolutions industrielles conduit à terme à la production de conditions sociales, psychologiques et culturelles favorables à la militance environnementaliste et à la mise en place de politiques publiques de protection de l'environnement. Inglehart et Welzel divisent ce processus en deux phases majeures. La première phase, dite de modernisation, voit à partir du $\mathrm{XIX}^{\mathrm{e}}$ siècle la mobilisation des masses, la laïcisation et le développement d'appareils d'État centralisés et impersonnels. Vient ensuite, après 1945, la phase post-matérialiste qui marque un approfondissement sans précédent de l'autonomie des individus ainsi 
que l'expression grandissante d'aspirations démocratiques. Inglehart et Welzel désignent ce long processus sous le nom de développement humain :

A massive body of cross-national data demonstrates that (1) socio-economic modernization, (2) a cultural shift toward rising emphasis on self-expression values, and (3) democratization are all components of a single underlying process: human development. The underlying theme of this process is the broadening of human choice. Socioeconomic modernization reduces the external constraints on human choice by increasing people's material, cognitive, and social resources. This brings growing mass emphasis on self-expression values, which in turn lead to growing public demands for civil and political liberties, gender equality, and responsive government, helping to establish and sustain the institutions best suited to maximize human choice-in a word, democracy. The core of the human development sequence is the expansion of human choice and autonomy. (2)

6 Même si tous les membres des sociétés dites post-matérialistes n'adoptent pas des valeurs post-matérialistes (visant à augmenter l'autonomie individuelle et la capacité des individus à faire des choix autonomes), loin s'en faut (Kidd 3-4), la distribution de plus en plus large de l'abondance matérielle dans les pays économiquement développés après la Seconde Guerre mondiale semble contribuer à l'éclosion d'attentes nouvelles en matière de politique publique. Sans adhérer au déterminisme économique, Inglehart et Welzel expliquent en somme que l'amélioration des conditions économiques favorise l'expression de valeurs post-matérialistes qui, in fine, se reflètent dans de nouvelles orientations politiques (134).

7 La transformation post-matérialiste touche de nombreux domaines, comme l'égalité entre les femmes et les hommes ou l'aspiration à un plus haut degré de représentation démocratique (Inglehart et Welzel 52-54). La question de la protection de l'environnement est l'un des principaux domaines concernés. Selon un schéma de pensée qui rappelle la fameuse pyramide de Maslow et ses réflexions autour de la motivation humaine (Maslow 80-106), Inglehart et Welzel affirment que la société d'abondance, qui garantit et assure les conditions de survie de l'immense majorité des individus, suscite chez nombre de ses membres une aspiration à une meilleure qualité de vie et à la recherche du bien-être :

Postindustrial modernization brings a fundamental shift in economic strategies, from maximizing material standards of living to maximizing well-being through life-style changes. The "quality of experience" replaces the quantity of commodities as the prime criterion for making a good living. The rise of self-expression values has changed the political agenda of postindustrial societies, challenging the emphasis on economic growth at any price by an increasing concern for environmental protection. (25)

8 Ainsi, c'est précisément parce que les membres des sociétés post-matérialistes ont les moyens matériels de se préoccuper davantage de leur qualité de vie et de la protection de la nature que l'environnementalisme politique fait son apparition à partir des années 1960 et 1970 dans plusieurs pays riches, comme l'Allemagne ou les États-Unis ${ }^{1}$.

9 L'historienne Lizabeth Cohen note que la société américaine fait l'expérience de la consommation de masse dès les années 1920, mais que la fin de la Seconde Guerre mondiale marque un tournant majeur dans l'histoire de la consommation aux ÉtatsUnis (22). Se développe alors, selon elle, "une république des consommateurs ». Le consensus keynésien d'après-guerre, qui considère la demande (consommation et investissements) comme le moteur principal de la croissance, place le consommateur américain au cœur des préoccupations gouvernementales : 
Wherever one looked in the aftermath of war, one found a vision of postwar America where the general good would be best served not by frugality or even moderation, but by individuals, pursuing personal wants in a flourishing mass competition marketplace. Only fear of inflation, as prices skyrocketed after the lifting of price controls and then again with the Korean War, and occasional business concerns over the nation's low savings rate cautioned any restraint. (L. Cohen 121)

10 Si la pauvreté ne disparaît pas du paysage américain, il est indéniable que le niveau de vie moyen augmente considérablement et à un rythme soutenu, et ce, quelques années seulement après la Grande Dépression et le second conflit mondial.

\section{David Brower et l'environnementalisme de la société d'abondance}

L'historien Samuel P. Hays a démontré que la distribution de plus en plus large de l'abondance matérielle et les aspirations consuméristes qui l'accompagnent ont joué un rôle décisif dans l'avènement du mouvement environnementaliste contemporain aux États-Unis, qui connaît son âge d'or dans les années 1960 et 1970. Hays différencie le conservationnisme productiviste du début du $\mathrm{xx}^{\mathrm{e}}$ siècle, né de l'alliance entre Theodore Roosevelt et Gifford Pinchot, de l'environnementalisme hédoniste et consumériste des années 1960 et 1970 dont Brower est l'un des protagonistes : "Conservation was an aspect of the history of production that stressed efficiency, whereas environment was a part of the history of consumption, that stressed new aspects of the American standard of living » (1993 13).

12 Hays perçoit le mouvement environnementaliste d'après-guerre comme un phénomène principalement urbain, adossé à de plus fortes attentes en matière de santé et de bienêtre (51). Par environnementalisme hédoniste et consumériste, il faut donc entendre ici l'apparition d'exigences grandissantes en matière de qualité de l'air et de l'eau, d'accès aux loisirs en plein air ou d'accès à la nature, que ce soit dans des parcs urbains ou dans des zones plus vastes protégées par les pouvoirs publics.

13 Ces aspirations nouvelles conduisent les Démocrates et les Républicains au Congrès à voter de nombreuses lois de régulation environnementale et de protection de la nature (Clean Air Acts à partir de 1963, Clean Water Acts à partir de 1960, Wilderness Act en 1964, Endangered Species Act en 1973 etc.) cependant que le président Richard Nixon crée en 1970 l'EPA (Environmental Protection Agency) dont la mission est de faire respecter les normes environnementales dans l'ensemble du pays. Au même moment, les organisations environnementalistes connaissent une expansion sans précédent, dont le succès inattendu de la première journée de la Terre en 1970 est le point d'orgue (Rome).

14 Sans utiliser la terminologie d'Inglehart et Welzel, l'homme politique Stewart Udall avait appelé dès 1963 à la nécessaire transition de la société américaine vers des valeurs et des attentes post-matérialistes :

One of the paradoxes of American society is that while our economic standard of living has become the envy of the world, our environmental standard has steadily declined. We are better housed, better nourished, and better entertained, but we are not better prepared to inherit the earth or to carry on the pursuit of happiness. (189) 

guerre ne va pas sans soulever d'étonnants paradoxes. Ainsi de l'appétence grandissante du peuple américain pour les randonnées et les séjours dans les parcs nationaux et les wilderness areas : elle garantit la pérennité de la protection fédérale des zones concernées, mais en menace l'intégrité en transformant ces espaces en objets de consommation de masse (Turner 469). Après 1945, des cadres du Sierra Club, dont David Brower, s'inquiètent par exemple des effets délétères du tourisme de masse sur les parcs nationaux (Brower, 1945). Cette situation paradoxale traduit parfaitement les tensions qui parcourent le mouvement environnementaliste contemporain que Brower a contribué à faire fructifier.

16 À la fin de la Seconde Guerre mondiale, David Brower est nommé directeur exécutif du Sierra Club. Il devait par la suite devenir l'un des militants les plus influents de la cause environnementaliste aux États-Unis. En quelques années, il transforme la modeste organisation fondée en 1892 par John Muir en un acteur collectif reconnu du monde associatif et politique. Il élargit l'éventail des sujets traités (pollution, santé publique, questions énergétiques etc.) et fait passer le nombre de membres du Club de 7000 en 1952 à 78000 en 1968 (M.P. Cohen 275). Afin d'accroittre la visibilité et la popularité du Sierra Club, Brower perpétue la tradition inaugurée au xIX ${ }^{e}$ siècle par les peintres, nature writers et photographes, en publiant de nombreux livres de photographies (coffee-table books) et en réalisant des documentaires à la gloire des paysages américains. Comme l'a montré François Brunet, la peinture et la photographie ont rempli très tôt une fonction cruciale dans la propagation du message préservationniste aux États-Unis (Brunet).

Brower se distingue aussi en opposant une résistance redoutablement efficace au Bureau of Reclamation, agence fédérale dont la mission est de construire des barrages, notamment dans les États de l'Ouest. Dans le cadre d'un grand projet d'irrigation des terres arides de l'Ouest américain (le Colorado River Storage Project), le Bureau of Reclamation entendait construire un barrage dans un lieu appelé Echo Park, à l'intérieur du Dinosaur National Monument. David Brower mena avec d'autres une campagne acharnée qui aboutit à l'annulation du projet en 1956. Brower et le Sierra Club menèrent ensuite une autre bataille victorieuse contre un projet de barrage qui aurait submergé une partie du Grand Canyon (Gottlieb 41-43). Club. Elle lui permet de s'affirmer comme un solide rempart face aux menaces qui pèsent sur les paysages majestueux de l'Ouest américain. En 1966, Brower demande : "Are we to maintain unimpaired some pieces of superlative landscape for their scenic, scientific, and recreational purposes, or are we going to use all our land for exploitative uses? " (Brower 1966). Ce refus de l'exploitation matérielle généralisée du vivant façonne la démarche militante de Brower qui contribue aussi à la création de Redwoods National Park et de North Cascades National Park, ou encore à la préservation de Point Reyes en Californie (Collomb 119). Il rejoint ainsi John Muir au panthéon des grands préservationnistes américains.

Il crée par la suite Friends of the Earth et la League of Conservation Voters en 1969, puis l'Earth Island Institute en 1982, et participe dans le même temps à la judiciarisation et à l'internationalisation du mouvement. La trajectoire de David Brower épouse donc parfaitement celle de l'environnementalisme américain contemporain puisqu'il contribue à la massification du mouvement, à l'accroissement de sa visibilité et à la 
diversification de ses préoccupations et de ses objectifs. Il fait partie de ceux qui parviennent à exploiter l'importance grandissante des valeurs post-matérialistes durant l'après-guerre et à les convertir en normes et lois environnementales. Certes, le bilan de Brower est à bien des égards remarquable, mais il est utile d'étudier la vulnérabilité d'une carrière dont le succès menace les acquis. De nombreux Américains suivent Brower parce qu'ils ont atteint un haut degré de confort matériel. Cependant, il est loin d'être certain qu'ils continuent de le suivre dès lors que ce confort est remis en cause, car c'est ce confort qui a permis l'éclosion d'un public sensible aux revendications environnementalistes.

\section{Rhétorique de l'austérité et société d'abondance}

Le militant qui bénéficie des conditions de la société d'abondance pour faire prospérer le mouvement environnementaliste est aussi celui qui livre un réquisitoire sans équivoque contre la croissance économique et la société de consommation. Brower dit commencer à remettre en cause le culte de la croissance au début des années 1960 (David R. Brower 315). Il a été influencé par le penseur de la convivialité Ivan Illich ${ }^{2}$ ainsi que par des économistes hétérodoxes comme E.F. Schumacher et Herman Daly. Dans l'esprit de Brower, les crises environnementales sont directement imputables à l'enrichissement des populations des économies développées : selon lui, "the great threat to the global environment is affluence » (1991 a). Il réserve d'ailleurs ses critiques les plus dures à la société américaine, plus énergivore et consommatrice que toutes les autres (Brower, 1970).

21 Son argumentaire repose sur l'idée qu'il est physiquement impossible pour une économie de croître indéfiniment dans un monde fini :

It will become clear in all Man's activities that a finite earth imposes limits-and is

likely to impose them harshly if Man does not himself impose them rationally first.

We will be rational enough to impose limits ourselves, and to impose them before

our numbers and desires have obliterated our heritage. (1971)

$\mathrm{Au}$ moment même où le monde occidental traverse une période exceptionnelle d'expansion économique, Brower exhorte ses contemporains à soumettre la pensée économique aux savoirs biologiques et physiques, comme devait le préconiser bien plus tard le philosophe J. Baird Callicott : «The human economy is a subset of the economy of nature " (62). Selon la vision de Brower, le développement économique est voué à s'inscrire à plus ou moins brève échéance dans les limites physiques qui le soustendent. Il n'est, certes, pas le seul à défendre cette position, mais il joue un rôle important dans sa diffusion.

Le réquisitoire de Brower comporte également une dimension esthétique. L'augmentation soutenue du PIB après 1945 ne dit rien, selon lui, de l'appauvrissement et de l'enlaidissement du monde qu'elle provoque :

We read that magnificently amorphous cliché "the greatest good for the greatest number" backwards-and end up with programs in which the greatest number can wipe out the greatest good, in which progress is measured by the number of things it wipes out which made life most worthwhile, by the number of things we collect rather than the number we understand, and by the number of acres added to the tax rolls but lost to society. (1964) 

d'alarme devant l'expansion démographique vertigineuse que le monde connaît au cours $\mathrm{du} \mathrm{xx}^{\mathrm{e}}$ siècle. Qu'il suffise de rappeler que Brower est celui qui convainc le biologiste Paul Ehrlich de publier son célèbre pamphlet malthusien The Population Bomb en 1968 et qu'il signe la préface de la première édition. À l'heure du baby-boom, Brower propose que les familles de plus de deux enfants soient taxées (McPhee 42). Il est évident dans son esprit que destruction environnementale et expansion démographique ont partie liée :

We fight the earth with every technical and economic tool that we can brandish. We are addicted to unreasonable growth in demands for nonrenewable resources, we obliterate renewable resources, and we insist upon exponential growth in our own numbers, despite having long ago passed the people limit. We speed euphorically on, borrowing irresponsibly from our children and theirs. (Brower, 1983)

Paul Ehrlich répète avec Brower que les politiques de protection de l'environnement sont vouées à l'échec aussi longtemps que l'espèce humaine continuera de croître (Ehrlich 44).

Afin de résoudre cette crise démographique et environnementale, Brower propose une limitation draconienne de la société de consommation à travers un éloge des limites et de la frugalité. Selon cette vision, l'opulence matérielle exceptionnelle dont jouissent les classes moyennes des économies développées depuis le milieu du $\mathrm{xx}^{\mathrm{e}}$ siècle ne pourra tout simplement pas être maintenue sans entraîner un irrémédiable 
délabrement du monde (1974). D'où l'espoir que fait naitre en lui le premier choc pétrolier de 1973 qu'il interprète à tort comme le début de la fin de la croissance (Brower, 1977-1982). Un an plus tôt, il s'était déjà montré enthousiaste à la lecture du rapport du Club de Rome dont les auteurs avaient insisté sur les " possibilités limitées » de la Terre et l'impossibilité de " la croissance exponentielle dans un système complexe et fermé » (Meadows 197). La nouvelle donne malthusienne qui semble se dessiner dans les années 1970 permet à Brower d'envisager une humanité qui, par la force des circonstances, deviendrait moins nombreuse, moins matérialiste, plus frugale et mènerait une vie plus saine. Dans un registre très similaire, Ivan Illich appelle à cette époque ses contemporains à «reconnaître l'existence d'échelles et de limites naturelles" qu'il convient de ne pas franchir afin de parvenir à une société conviviale (Illich 13). Celle-ci est, selon Illich, «la société où l'homme contrôle l'outil », où les individus préservent leur autonomie en s'affranchissant des grands systèmes hiérarchisés dépendant d'élites spécialisées (13). Brower croit alors que ce moment est venu. Il convient de préciser qu'il n'est pas à l'origine de ce point de vue, mais qu'il le défend bruyamment en politique et dans la société civile par ses actions, ses publications et ses interventions publiques.

31 Il s'appuie notamment sur les travaux que l'économiste Herman Daly a consacrés à l'économie de la stabilité. Selon Daly, la question fondamentale est celle du conflit entre la finitude et la croissance illimitée. Il s'inquiète de l'incapacité de la plupart des économistes à percevoir l'impasse physique de la croissance infinie et propose un horizon nouveau pour les sciences économiques, qu'il nomme économie de la stabilité (steady-state economy):

By "steady state" is meant a constant stock of physical wealth (capital) and a constant stock of people (population). Naturally these stocks do not remain constant by themselves. People die, and wealth is physically consumed-that is, worn out, depreciated. Therefore the stocks must be maintained by a rate of inflow (birth, production) equal to the rate of outflow (death, consumption). But this equality may obtain, and stocks remain constant, with a high rate of throughput (equal to the rate of inflow and the rate of outflow), or with a low rate. (Daly 14)

Loin d'être positif, le culte de la croissance est vu par Daly et Brower comme une pathologie de l'âge moderne. Brower propage sans relâche cette idée, également défendue par Ivan Illich, qui souhaite l'avènement d'un «équilibre postindustriel » (Illich 9).

Dans les années 1980, alors que le concept de développement durable commence à faire florès suite à la publication du rapport Brundtland de 1987 (World Commission on Environment and Development), Brower s'empresse d'exprimer son scepticisme :

Sustainable development was a contradiction in terms; growth and development mean further industrializing and thus exacerbating the environmental crisis. Trying to correct a problem by repeating the cause is like trying to sober up on martinis and won't work. Yes there is a nice way to define sustainable development, but there are no examples of it in industrial society. (Brower, 1992)

Malgré son admiration pour Gro Brundtland, femme d'État norvégienne et principale rédactrice du rapport de la Commission mondiale sur l'environnement et le développement de l'ONU, Brower conçoit le développement durable comme une nouvelle itération de la négation moderne des limites physiques du monde. Il aimerait pour sa part que ses contemporains cessent de faire de la croissance économique une priorité et reconnaissent les mérites et la nécessité de l'économie de la stabilité. Il 
estime que le développement durable risque d'entretenir l'opinion publique dans l'illusion d'une croissance verte qui parviendrait à concilier l'abondance matérielle exceptionnelle de la seconde moitié du $\mathrm{xx}^{\mathrm{e}}$ siècle et la préservation des écosystèmes et des espèces menacées. Cette illusion est pernicieuse, selon lui, car elle retarde une nécessaire prise de conscience des opinions des pays économiquement développés (Brower, 1992).

On mesure ici la vulnérabilité de sa démarche. Brower prône en effet la frugalité, l'austérité et la retenue au cœur même d'une extraordinaire période d'enrichissement matériel qui permet au souci de la protection de l'environnement de trouver une expression politique concrète. Or, si ses mises en garde trouvent un écho, c'est précisément parce que la majorité des Américains ont rejoint les rangs de la classe moyenne et qu'ils aspirent désormais à une amélioration de leur cadre de vie. Mais Brower adopte souvent dans ses écrits et interventions publiques une tonalité culpabilisante et moralisante. À mi-chemin entre Hobbes et Malthus, sa prose tranche avec l'hédonisme et l'optimisme de ses contemporains. Dans les années 1960 comme après la crise énergétique des années 1970, il fustige une humanité gloutonne, vorace et irresponsable: "Like an individual overdependent on alcohol, a society that has overindulged on oil has a difficult transition to face " (1979). En 1969, il affirme, lors d'une intervention publique, que ce sont les consommateurs dans leur ensemble qui portent la lourde responsabilité de la crise environnementale en cours : «Who buys the items that are polluting our environment? Everybody who buys them is an enemy of the environment. We do; and I am not going to call you all guilty. My family is as guilty as anyone, but this has got to stop. You are the villain, and I am the villain " (Brower, 1969). Tout en se défendant de porter un jugement moral, il se montre souvent particulièrement véhément à l'encontre de ses concitoyens américains et de leur mode de vie (1970).

On retrouve des échos de la rhétorique de Brower dans le célèbre discours que le président Jimmy Carter prononce en 1979 au sujet de la crise énergétique. Carter y avait notamment souligné l'influence délétère du consumérisme sur la morale du peuple américain :

In a nation that was proud of hard work, strong families, close-knit communities, and our faith in God, too many of us now tend to worship self-indulgence and consumption. Human destiny is no longer defined by what one does, but what one owns. But we've discovered that owning things and consuming things does not satisfy our longing for meaning. We've learned that piling up material goods cannot fill the emptiness of lives which have no confidence or purpose. (Carter 113)

Dans un registre similaire, Brower met en garde ses contemporains : " What we haven't discovered even now, is how to leave hubris at the door. There is still an overdose of pride in our species» (2000 19). De fait, à travers sa critique de l'hubris moderne, Brower remet en cause le projet cartésien de maitrise du vivant qui a rendu possible la révolution industrielle et in fine la société de consommation. Selon cette vision, qui repose sur un désenchantement radical du monde, l'humanité est en mesure, grâce à la science et à ses applications techniques, de maittriser et de transformer la nature afin de servir son bien-être. Brower perçoit dans cette démarche un refus dangereux de s'adapter au monde physique duquel dépend l'espèce humaine et d'en accepter les limites.

L'implacable réquisitoire de Brower contre les externalités négatives de la croissance et de la consommation de masse rencontre de nombreux échos dans une société qui, nous 
l'avons vu, est prête à l'entendre et qui constate, à travers la médiatisation de catastrophes environnementales et la publication d'ouvrage de sensibilisation comme le Silent Spring de Rachel Carson, que la diffusion de la prospérité économique comporte parfois un coût environnemental et sanitaire exorbitant (Gottlieb 83-86). Il convient cependant de noter que Brower fait très rarement référence aux bienfaits des tendances qu'il réprouve. Le fondateur de Friends of the Earth reconnaît la réalité de l'abondance matérielle, puisqu'il la combat, mais il ne dit rien de ses avantages. Il avoue lui-même peiner à reconnaître l'apport positif de la recherche scientifique et de ses applications techniques. Ainsi du lac Powell, ce réservoir retenu par le barrage de Glen Canyon à la frontière de l'Arizona et de l'Utah : «When I look across Lake Powell, I do not see the genius of human creativity. I can only think of the human addiction to destroying wild places and the species, besides our own, which inhabit them » (1997). On touche ici aux limites de la démarche de Brower car, si ses contemporains sont désireux de protéger l'environnement, ils n'ont pour la plupart aucune envie d'abandonner l'opulence et les conforts acquis après 1945.

\section{Conclusion : la résilience du consensus consumériste}

La carrière de David Brower résume le bilan de l'environnementalisme américain d'après-guerre dont la visibilité et l'influence grandissent considérablement, mais qui s'avère incapable de réorienter le système socio-économique de manière radicale. La réaction de l'opinion publique américaine à la crise énergétique des années 1970 en offre sans doute l'une des meilleures illustrations. Brower entend profiter de ce moment malthusien pour détourner ses concitoyens du culte de la croissance et les convaincre de la nécessité impérieuse d'un mode de vie plus frugal. Selon l'historien David E. Nye, c'est l'inverse qui se produit. Bien loin de provoquer une prise de conscience générale, la crise énergétique est vécue par de nombreux Américains comme une menace pour leur mode de vie, mais leurs attentes et leurs habitudes ne subissent pas de changement notable (228-237). Samuel P. Hays ajoute que la pénurie de carburant et les inquiétudes quant à l'approvisionnement énergétique de l'économie américaine réduisent l'influence des environnementalistes (1993 411-413), comme si l'attrait de leurs propositions ne pouvait résister à la perspective d'un recul de l'opulence. La parenthèse malthusienne se referma d'ailleurs rapidement au cours des années 1980 (Yergin 232).

L'élection de Ronald Reagan à la présidence des États-Unis en 1980 marque la fin de cet âge d'or de l'environnementalisme américain. La nouvelle administration perçoit l'appareil de régulation environnementale comme une entrave au bon fonctionnement des marchés et à la création de richesse. Les budgets alloués à la protection de l'environnement ainsi que la marge de manœuvre accordée aux agences fédérales de protection de l'environnement sont considérablement réduits (Short 135). En réaction, les grandes organisations environnementalistes se mobilisent et enregistrent une forte augmentation du nombre de leurs membres et sympathisants (Shabecoff 229-230). L'attractivité du mouvement environnementaliste augmente au moment même où le culte de la croissance économique atteint son paroxysme. Les environnementalistes américains sont alors en mesure de mobiliser un nombre considérable de leurs concitoyens, mais il leur devient presque impossible de faire adopter de nouvelles régulations environnementales et d'étoffer l'appareil fédéral de protection de 
l'environnement. Le mouvement prospère donc, mais le consensus bipartisan de la décennie précédente a disparu.

Le sociologue Robert J. Brulle a souligné les limites d'un mouvement environnementaliste contraint de s'adapter aux exigences du marché et au cadre fixé par l'État moderne :

Instrumental reason forms the dominant rhetoric of legitimization of both market and state actions. The atrophy of the public sphere seals off the lifeworld from providing input into the administrative state and the market. This creates a social order dominated by the narrow instrumental rationality that defines the imperatives of the state and the market. Fulfilling the instrumental imperatives of these two institutions forms the dominant legitimization scheme for social action, unrestrained by moral or aesthetic rationality. (38)

Il est indéniable que les environnementalistes obtiennent des résultats probants, à travers la création de zones protégées et la mise en place de normes sanitaires et environnementales, mais ces progrès sont voués à demeurer très limités dans la mesure où leur stratégie revient à s'inscrire dans un cadre déterminé par la recherche de la croissance et par la domination humaine du vivant au moyen des sciences et des techniques, et non à remettre en cause la légitimité même de ce cadre.

\section{BIBLIOGRAPHIE}

Sources citées

BROWER, David. « How to Kill a Wilderness ». Sierra Club Bulletin, n 30, 1945. David Ross Brower Papers, BANC MSS 79/9 c, CTN4:85.

---. Journal, octobre-novembre 1962. David Ross Brower Papers, BANC MSS 79/9 c, BOX18:9.

---. « The Irreplaceables, Foundations, and Conventional Heresy ». Sierra Club Bulletin, $\mathrm{n}^{\circ}$ 49, 1964, David Ross Brower Papers, BANC MSS 79/9 c, CTN5:17.

---. « Dams in the Grand Canyon? No. The Whole Grand Canyon Should Be Preserved ». The Book of Knowledge Annals, 1966. David Ross Brower Papers, BANC MSS 79/9 c, CTN5:21.

---. « Cities and our Natural Environment: Conditions for Peaceful Coexistence ». Colorado College Symposium. «The City », 11 janvier 1967, Colorado Springs, Colorado. Discours. David Ross Brower Papers, BANC MSS 79/9 c, CTN9:65.

---. « Talk, Conference on the Population Explosion ». Airlie House, Decatur, 21 novembre 1969, Decatur, Georgia. Discours. David Ross Brower Papers, BANC MSS 79/9 c, CTN9:75.

---. « The Search for an Environmental Perspective ». 1970. Discours. David Ross Brower Papers, BANC MSS 79/9 c, CTN5:29.

---. Preface to Eryri: The Mountains of Longing. 1971. David Ross Brower Papers, BANC MSS 79/9 c, CTN4:4.

---. « Life in Post-Industrial America ». 1974. Discours. David Ross Brower Papers, BANC MSS 79/9 c CTN5:42. 
---. Miscellaneous articles Not Man Apart. 1977-1982. David Ross Brower Papers, BANC MSS 79/9 c, CTN6:28.

---. Foreword to Pathways. 1979. David Ross Brower Papers, BANC MSS 79/9 c, CTN4:22.

---. Lettre adressée à Dennis Paulson. 23 novembre 1983. David Ross Brower Papers, BANC MSS 79/9

c, CTN4:27.

---. « Healing Time on Earth ». 6 octobre 1991. Discours. David Ross Brower Papers, BANC MSS 79/9

c, CTN10:56.

---. Work in Progress. Salt Lake City : Peregrine Smith, 1991.

---. « Fiddling While the Earth Burns ». Earth Island Journal, vol. 7, n 3, 1992, p. 13-14. David Ross Brower Papers, BANC MSS 79/9 c, CTN5:104.

---. Foreword to Science Under Siege. 1997. David Ross Brower Papers, BANC MSS 79/9 c, CTN4:51.

---. Let the Mountains Talk, Let the Rivers Run. 1995. Gabriola Island : New Society Publishers, 2000.

BRULLE, Robert J. Agency, Democracy, and Nature: The US Environmental Movement from a Critical Theory Perspective. Cambridge : MIT Press, 2000.

BRUNET, François. « Photographie et écologie aux États-Unis : l'image à contre-emploi ». Transatlantica, $n^{\circ} 1 \mid 2017$, journals.openedition.org/transatlantica/8878. Page consultée le 26 mars 2019.

CALLICOTT, J. Baird. « 5 Questions ». Sustainability Ethics. Dir. Ryne Raffaelle, Wade Robinson et Evan Selinger. Copenhague : Automatic Press/VIP, 2010, p. 57-70.

CARTER, Jimmy. « Energy and National Goals ». Jimmy Carter and the Energy Crisis of the 1970s: The Crisis of Confidence Speech of July 15, 1979. Dir. Daniel Horowitz. Boston : Bedford/Saint Martin's, 2005, p. 112-114.

COHEN, Lizabeth. A Consumers' Republic: The Politics of Mass Consumption in Postwar America. 2003. New York : Vintage Books, 2004.

COHEN, Michael P. The History of the Sierra Club. San Francisco : Sierra Club Books, 1988.

COLLOMB, Jean-Daniel. Une Histoire de la radicalité environnementale aux États-Unis. Pessac : Presses universitaires de Bordeaux, 2018.

DALY, Herman E. « Introduction ». Toward a Steady-State Economy. Dir. Herman E. Daly. San Francisco : W.H. Freeman, 1973, p. 1-29.

David R. Brower-Environmental Activist, Publicist and Prophet: An Interview Conducted by Susan Schrepfer, 1974-1978. Berkeley : Regional Oral History Office, 1980.

EHRLICH, Paul R. The Population Bomb. 1968. Cutchogue : Bucaneer Books, 1971.

ELLUL, Jacques. Le Bluff technologique. 1988. Paris : Hachette, 2004.

GOTTLIEB, Robert. Forcing the Spring: The Transformation of the US Environmental Movement. Washington, DC : Island Press, 1993.

HAYS, Samuel P. Conservation and the Gospel of Efficiency: The Progressive Conservation Movement, 1890-1920. 1959. Pittsburgh : University of Pittsburgh Press, 1999.

---. Beauty, Health, and Permanence: Environmental Politics in the United States, 1955-1985. 1987. Cambridge : Cambridge University Press, 1993.

ILLICH, Ivan. La Convivialité. 1973. Paris : Seuil, 2003. 
INGLEHART, Ronald, et Christian WELZEL. Modernization, Cultural Change, and Democracy. 2005. Cambridge : Cambridge University Press, 2010.

KIDD, Quentin, et Aïe-Rie LEE. « Postmaterialist Values and the Environment: A Critique and Reappraisal ». Social Science Quarterly, vol. 78, n 1, 1997, p. 1-15.

MASLOW, Abraham H. Motivation and Personality. New York : Harper, 1954.

MCPHEE, John. Encounters with the Archdruid. 1971. New York : Farrar, Strauss, and Giroux, 1992.

MEADOWS, Donella H., Dennis L. MEADOWS et Jørgen RANDERS. Halte à la croissance ?. Traduit de l'anglais par Janine Delaunay. Paris : Fayard, 1972.

NYE, David E. Consuming Power: A Social History of American Energies. Cambridge : MIT Press, 1998.

ROME, Adam. The Genius of Earth Day. New York : Hill and Wang, 2013.

SCHUMACHER E.F. Small is beautiful : une société à la mesure de l'homme. 1973. Traduit de l'anglais par Danielle Day, William Day, Marie-Claude Florentin et Patrick Jeanjean. Paris : Seuil, 1978.

SHABECOFF, Philip. A Fierce Green Fire: The American Environmental Movement. 1993. New York : Hill and Wang, 1994.

SHORT, C. Brand. Ronald Reagan and the Public Lands: America's Conservation Debate 1979-1984. College Station : Texas A\&M University Press, 1989.

TURNER, James Morton. « From Woodcraft to "Leave No Trace": Wilderness, Consumerism, and Environmentalism in Twentieth-century America ». Environmental History, vol. 7, n 3, 2002 , p. $462-484$.

TURNER, Tom. David Brower: The Making of the Environmental Movement. Oakland : University of California Press, 2015.

UDALL, Stewart L. The Quiet Crisis. 1963. Londres : Forgotten Books, 2015.

WORLD COMMISSION ON ENVIRONMENT AND DEVELOPMENT. Our Common Future. Oxford : Oxford University Press, 1987.

YERGIN, David. The Quest: Energy, Security, and the Remaking of the Modern World. New York : Allen Lane, 2011.

\section{NOTES}

1. Il est ici question du mouvement environnementaliste contemporain, qui a des prédécesseurs, bien que ceux-ci ne se désignent pas comme environnementalistes. On pense aux préservationnistes qui s'activent dès la seconde moitié $d u$ XIX ${ }^{\mathrm{e}}$ siècle en faveur des parcs nationaux puis, plus tard, des wildlife refuges et des wilderness areas. Il convient aussi de mentionner le conservationnisme utilitaire de Gifford Pinchot qui trouve son expression dans les forêts nationales et le United States Forest Service qui les gère dès 1905.

2. Né à Vienne en 1926, Ivan Illich est un penseur autodidacte qui publie en 1973 La convivialité. Cet ouvrage brosse le tableau d'une humanité moderne instrumentalisée par ses propres outils et entretient l'espoir d'une sortie de la société industrielle. Selon lui, la démesure de la technique moderne conduit à des dépassements de seuils qui, bien loin de rendre les activités humaines plus efficaces, leur donnent un tour contre-productif et nocif. 


\section{RÉSUMÉS}

Cet article porte sur la vulnérabilité intrinsèque de la militance environnementaliste aux ÉtatsUnis dans le contexte de la société d'abondance. Durant la période qui suit les deux guerres mondiales, le succès grandissant cette militance semble tenir pour une grande partie à la distribution sans précédent de la prospérité matérielle. Nombreux sont les environnementalistes américains qui, après 1945, mettent en garde leurs concitoyens quant aux externalités négatives de la croissance économique et de la consommation de masse, cherchant de fait à éradiquer un type d'organisation économique qui a permis aux thèses environnementalistes de progresser dans l'opinion publique. Le mouvement environnementaliste semble alors dépendre des conditions socio-économiques qu'il combat. Il s'agira donc d'interroger les contraintes qu'une telle tension impose à la militance environnementaliste : si la remise en cause de l'abondance matérielle aboutit, y aura-t-il encore une volonté de préserver l'environnement? Pour répondre à ces interrogations, le présent article se concentre sur le cas de David Ross Brower, grand artisan de la transformation du Sierra Club après-guerre et fondateur de Friends of the Earth, en s'appuyant sur ses archives rassemblées à la Bancroft Library de l'université de Californie à Berkeley. Quel discours Brower tient-il sur les conditions socio-économiques de son époque? Quelles sont les limites de son engagement militant dans un tel contexte?

This article addresses the vulnerability of American environmentalist discourse during the postWorld War II boom: while the growing success of the environmental movement in the United States can largely be attributed to an unprecedentedly large distribution of material wealth after 1945, many environmentalists had warned Americans against the negative effects of economic growth and mass consumption. Therefore, the success of the environmental movement depended on the very socioeconomic conditions against which it was protesting, which, from a strategic perspective, meant that the end of prosperity might also undermine public appetite for environmental protection. This article analyzes the constraints this tension imposes on environmental advocacy. It takes the works of the prominent environmentalist David Ross Brower as a case study in order to address the following questions: How did Brower deal with the socioeconomic conditions of the postwar years? What constraints did those conditions impose on his advocacy? The article uses material from Brower's papers that are held in the Bancroft Library at the University of California, Berkeley.

\section{INDEX}

Thèmes : Hors-thème

Keywords : consumerism, consumption, energy crisis, environmentalism, growth, Malthusianism, militancy, post-materialism, sustainable development

Mots-clés : consommation, consumérisme, crise énergétique, croissance, développement durable, environnementalisme, malthusianisme, militance, post-matérialisme

\section{AUTEUR}

\section{JEAN-DANIEL COLLOMB}

Université Jean Moulin (Lyon 3)

jean-daniel.collomb@univ-lyon3.fr 